\title{
Mythbusters in rowing medicine and physiotherapy: nine experts tackle five clinical conundrums
}

\author{
Fiona Wilson, ${ }^{1}$ Alison McGregor ${ }^{2}$
}

${ }^{1}$ Discipline of Physiotherapy, School of Medicine, Trinity College Dublin, Dublin, Ireland ${ }^{2}$ Department of Surgery and Cancer, Faculty of Medicine, Imperial College London, Charing Cross Hospital, London, UK

Correspondence to Dr Fiona Wilson, Discipline of Physiotherapy, School of Medicine, Trinity College Dublin, Trinity Centre For Health Sciences, St James's Hospital, Dublin 8, Ireland; wilsonf@tcd.ie

Accepted 17 September 2014

\section{INTERVIEW QUESTIONS Cardiac arrhythmias}

Dr Chris Milne: Medical Director, Rowing New Zealand

What is your experience as a team physician in dealing with insidious onset of cardiac disorders in rowers?

Any rower who presents with palpitations should be taken seriously, as these may be an indicator of significant cardiac arrhythmia. The many years of hard endurance training causes adaptive changes that are collectively known as 'the athlete's heart'. Sometimes these changes progress from normal adaptation to volume overload to unhealthy sequelae.

The most common arrhythmia seen in rowers is atrial fibrillation, and the longer the rower has been in heavy training, typically over 10 years, the more likely it is that they will develop this arrhythmia. The exact mechanism is unclear but it may be due to atrial enlargement with dilation and fibrosis plus a contribution from increased vagal tone and bradycardia.

The condition presented dramatically for Rob Waddell in the trials for the single berth at Beijing Olympics. In New Zealand, several athletes' rowing careers have been affected by atrial fibrillation or other arrhythmias. Nathan Cohen, gold medallist in the London 2012 Olympics, had to retire following several episodes of arrhythmia.

Can these conditions be detected by routine screening?

The history and physical examination occasionally provide clues. A resting ECG (recently introduced by Fédération Internationale des Sociétés d'Aviron (FISA) as a requirement at World Championships at Under-23 level (U23)) can provide additional clues to underlying cardiac disorders (eg, hypertrophic cardiomyopathy) and may prompt referral for other more advanced investigations, for example, echocardiography or electrophysiological studies. Liaison with a cardiologist who has an interest in these issues is recommended.

Rob Waddell: Athlete, Olympic Champion, single scull (Sydney 2000), $2 \times$ world champion.

What was your experience as an athlete with a cardiac abnormality and what challenges did you face in obtaining a diagnosis?

I was only 21 years old, preparing for international competition; I had already spent a year suffering from symptoms that only occurred in racing and would result in a large loss of power and shortage of breath. My common sensation was that it felt like I was suddenly rowing in mud.

As a young athlete, I was very confused and put the problem down to a number of potential causes -dehydration, overtraining, pain barrier, mental weakness, etc. I tried a wide range of solutions including several days' rest before major events and visiting sports psychologists. None of these made any noticeable difference and the effects were humiliating (often in crew situations) as well as a big dent in my confidence. I discussed what was happening casually with a cardiologist, who suggested it could be a heart problem requiring investigation. Testing induced an arrhythmia; results were conclusive and it was a huge relief to know that I had a 'real problem'.

Solutions, however, were not obvious. Ablation was in very early stages (1993). I worked hard on controlling it by reducing exposure to key factors that might set it off-fatigue, stress, alcohol, sleep deprivation, etc, which had some effect; but the real breakthrough came at a racing regatta in Australia. The problem reoccurred and one of the Australian Team doctors suggested the medication Tambocor (flecainide). At that point I had been put in the single scull because of my problem, and from 1996-2000, I relied heavily on flecainide. It took me several years to regain confidence in myself. Racing at that level is tough enough without extra complications. Flecainide worked very well right until the year of the Olympics in 2000 where, unfortunately, it just stopped working and episodes started again.

We went into investigation overdrive and learnt that for some strange reason, although my dosage had not changed (300 mg daily), I was not getting a therapeutic amount absorbed into my blood stream. In somewhat uncharted territory my dosage rose up to $600 \mathrm{mg}$ around competition-which resulted in just the right amount of flecainide in my system. I only did this in the week of competition. We scraped through the week of the Olympics but my cardiologist told me that if I ever wanted to race again I would need to have ablation surgery. I then retired from rowing and took up sailing and because of the large change in training (no big cardio) the problem drifted away and was not such an issue.

The problem only reared again when I decided to make a comeback to rowing and unfortunately had no problems until my critical trial race of the Olympic season when I experienced an episode $250 \mathrm{~m}$ into the final race. The following year I finally decided to have the ablation operation. The problem had become an everyday occurrence and so my motivation was not so much a sporting one, but more a general health and well-being one. Since the surgery in 2009, I have had three episodes, but no more. It has made a big difference to my general health and greatly prolonged my sailing career. 
Although I no longer compete at a high level in rowing, I feel part of the benefit has come from stopping rowing. The huge strain it places on the heart was a factor for my problem and I feel it would come back again should I push it to those extremes. I am very grateful to doctors including Dr Chris Milne for their help in diagnosing and dealing with the problem throughout my career. I would have desperately liked a couple more Olympic Gold medals! However, am very grateful for the one I have and know it only happened because of the good team of people I had assisting me along the way.

Dr Larissa Trease: Principal Medical Officer, Rowing Australia

What did you learn in your implementation of the FISA recommended cardiac screening protocol?

In response to the FISA compulsory cardiac screening for Junior World Championship competitors, Rowing Australia conducted an on-site screening service at our selection trials. Screening was compulsory for junior trialists and recommended for U23 and Senior athletes. Australia does not have any legislative requirement for cardiac or general medical screening prior to participation in competition, nor does government funding support this process.

In total we performed 185 ECGs and these were analysed using the Seattle Criteria (1) by a sports cardiologist. Twenty-six athletes required further investigation for 'type 2', or 'uncommon in sport changes' in their ECGs. They underwent echocardiogram on-site. Of this group, three athletes went on to require further follow-up, including two athletes who were referred to a genetic cardiologist for possible pathologies associated with sudden cardiac death.

What were the key lessons learnt from this process, which may assist team physicians in preparing their screening process?

The Seattle Criteria and the European Cardiology Society guidelines for ECG interpretation in the athlete will assist the clinician in identifying exercise-associated changes that are benign and do not require further investigation. Athletes with ECG changes that are not commonly seen as a result of exercise should be referred for further investigation and an opinion from a cardiologist with expertise and interest in endurance sports.

The timing of screening in the rowing year provides some challenges. Screening at the time of team selection means that those athletes requiring further investigation are selected, with the possibility that they may be unable to continue to compete after follow-up, disrupting crew preparation. Screening prior to selection would avoid this issue; however, this requires screening of much higher numbers of athletes, with a greater cost burden.

If screening, ensure it is performed properly. A lot is at stake and the misinterpretation of the ECG by a non-sports specialist can have many effects, including precluding participation by athletes in their chosen sport, difficulties with the athlete obtaining travel and life insurance, and considerable financial and time cost and concern.

A thorough consent process is important and needs to involve the parents of junior athletes. While the incidence of serious pathologies is low, our experience this year has demonstrated that one or two athletes may have their ability to participate in sport curtailed as a result of the findings of screening. Athletes and their families must understand prior to participating in screening this small, but real possibility.

Finally, the frequency of screening is yet to be determined by the FISA Medical Commission and this is a difficult area without clear guidance from the literature. There are multiple considerations in determining frequency, including the age of the athlete, the presence of previous normal screening results, the cost burden of repeated screening and the logistical challenges of screening in countries without state funded screening programmes.

\section{Back pain}

Mark Edgar: Physiotherapist, Head of Medical Services, GB Rowing.

Do athletes or medics misinterpret or have odd beliefs about back pain?

Most rowers train or race with some sort of discomfort, either from a structural injury or simply from training fatigue/ overuse. The idea of a 'back strain' and that the rowers will 'just train through it' is common. Unfortunately, some back injuries, such as lumbar spine annular wall loading, potentially leading to annular wall compromise are quite common. The initial stages of this problem may not be so painfully acute, however, repetitive strain on the poster annular wall resulting in creep, which leads to matrix breakdown probably makes the same structure vulnerable to failure if significant loading is subsequently applied. For example, long sessions on an ergo or water followed by heavy weights or heavy weights early in the morning/periods of sitting followed by rowing training.

In all of these scenarios the rower probably fails to appreciate that normal daily activities also have significant effect on the loading of the posterior annular wall. Most athletes and clinicians misinterpret the length of time that normal connective tissue takes to repair before rehabilitating to normal extensibility. This may include appropriate restoration of contractile tissue repair and restoration of normal neurological conduction/ conductivity.

What are your thoughts on fear avoidant behaviours in athletes-do you see them as much as you do in the normal population?

There is more avoidant (denial) behaviour within the athletic population especially if there are extrinsic factors such as selection pressures, regatta timetables, etc. Avoidance may be due to poor diagnosis and misinterpretation of signs and symptoms right up to the point where denial of a potentially serious issue is tolerated. Group mentality-squad pressures and the suggestion that an athlete 'will just row it off' or an athlete 'will just row through it'-exacerbates the issue. The general population may be more ready to take time to allow the body to resolve and does not have the pressure of a defined training programme, selection criteria or peer pressures to contend with. The athlete may seek help quickly but may have unrealistic expectations of their ability to rejoin the full training programme and squad environment.

Craig Newlands: Lead Physiotherapist, Rowing New Zealand

Fear avoidant behaviours are not unheard of in rowing athletes. There are instances when the mental barrier of returning to training on the water is as much of an issue as the physical injury. This seems more common in those athletes who have had a previous low back injury that has affected their training for a long period. Those athletes who have had a sudden onset of pain during a rowing session are also often more reluctant to return to training than, say, those who have had a gradual onset of symptoms, or first felt their pain doing another activity. Therefore, it is important to maintain some form of lowintensity rowing-specific training, if possible, early on in an athlete's rehabilitation in order to reduce the mental barrier of returning to on water training. This will also help minimise the deconditioning effect of not training.

Managing the return to training with an athlete's fear of reinjury balanced with their desire to keep training; the external 
pressure from the rest of the crew, the coach and management; as well as the stage of healing of the injured structure, can be very difficult.

Kellie Wilkie: Lead Physiotherapist, Rowing Australia

How do you manage low back pain in the lead up to elite competition?

Within the Rowing Australia medical and physical therapy network we emphasise that early intervention for low back pain is crucial but also advocate a response to management that is not 'alarmist'. We want athletes to have confidence that they can approach us and know that we will be focused on case managing them to ensure the best performance outcome for competition. Any medical personnel travelling with a rowing team knows that this is difficult but is significantly aided by an honest athlete who does not try to 'tough it out'.

The low back pain literature informs us that in the presence of pain, muscle recruitment patterns are altered. There are many alterations reported in the literature and we agree that clinically there are many patterns of movement dysfunction that present in the presence of low back pain in rowers. We ensure that the athlete understands that the presence of pain is likely to change the way they recruit muscles and this has a direct impact on performance. Our main aim is to reduce or control pain primarily for rowing but also for all activities of daily living. We do this with a combination of manual therapy, medication, load alteration and have also started introducing pain education and mindfulness interventions.

We have had recent successes in a therapy approach that is not 'alarmist'. We educate athletes about their injury and pain. We often find that the description that athletes verbalise about what their injury may look like, or the pathology as they understand it, is quite dramatic and simple pain education can reduce their fear. We ensure athletes know about how stress can influence pain sensitivity and that this is often amplified coming into competition. We work with them to establish techniques for self-regulating muscle tone via breathing techniques. We are beginning to incorporate mindfulness skills to assist management and improve sleep patterns that are often affected due to pain and stress.

The challenge is finding the balance to ensure athletes tell you about their pain in the first instance while ensuring that they and their coach keep calm. This improves with athlete maturity and is certainly easier to manage in reoccurrence if the athlete is well informed about pain and stress from a previous episode.

\section{RIB STRESS FRACTURES}

Anders Vinther: Physiotherapist, Rowing Denmark (1999-2001), Assistant Professor Lund University

What is the best way to manage a rib stress fracture?

Normally, a symptom-dependent approach is taken involving a period of complete rest-until deep breathing and increased ventilation is pain free-followed by a period of non-rowing exercise-that is, stationary bicycling — and eventually a gradual return to rowing. During this period, analgaesics, electrotherapy, icing, thoracic spine mobilisation and proprioceptive strapping with tape can treat pain and discomfort. The use of nonsteroidal anti-inflammatory drugs should be avoided as they have been indicated to slow down fracture healing. Typically, rowing training is gradually resumed after 2-6 weeks of relative rest. The loading must be very carefully reintroduced. Biomechanical studies suggest that loading during on-water rowing is lower than during dynamic ergometer rowing and highest during stationary ergometer rowing. This knowledge can be used during the gradual return to rowing. Similarly, training volume and training intensity should be increased gradually. Recurrent symptoms should lead to rest and resumption of training at a lower load.

A more aggressive management strategy, involving no or a very short period of rest and intensified treatment of pain including constant strapping, has been used in rowers in the lead-up to or during major competitions. This approach is reported to compromise pain relief to maintain performance levels of the crew; this is in conflict with the general practice in stress fracture management in athletes where rule number one is to modify activity (symptom-dependent approach). Anecdotal reports of non-union of rib stress fractures in elite rowers do exist. Consequently, great care should be taken to make sure that an adequate period of rest is introduced as soon as possible to avoid this potentially career-ending injury. The period of rest needed to allow sufficient healing is very likely to be prolonged if the aggravating activity (rowing) is continued despite symptoms of rib stress fracture.

A very important aspect of stress fracture management in general is to assess and modify potential risk factors such as low energy availability, menstrual disorders, calcium or vitamin D deficits, etc, to avoid recurrent stress fractures.

\section{NUTRITION}

Dr Gary Slater: Senior lecturer, University of the Sunshine Coast, Australia

What is the best recovery strategy for a lightweight rower after weigh-in?

Similar to athletes from other weight category sports, lightweight rowers are reported to use acute weight loss strategies prior to competition, with few competitors 'considered natural lightweights'. Among lightweight rowers, body mass may reduce by as much as $8 \%$ between the preseason and competition periods, suggesting that few lightweight rowers are natural 'lightweights'. At least half of the body mass lost by lightweight rowers during training can occur in the final week before a regatta, suggesting that both acute (achieved over a 24-72 h period) and chronic (achieved over a period of weeks) weight loss strategies are employed.

Popular practices include increase in training volume, food restriction, active and/or passive sweat production and fluid restriction; the majority of athletes acknowledge the use of at least two weight loss practices. The impact of acute weight loss strategies on performance remains an issue of conjecture. Activities demanding high instantaneous power output and absolute strength are less likely to be influenced by acute weight loss, but performance in activities that demand high aerobic and anaerobic capacities is typically compromised. However, the latter effect may be diminished when there is a recovery period between weigh-in and competition; lightweight rowers are required to weigh-in not less than $1 \mathrm{~h}$ and no more than $2 \mathrm{~h}$ before each race during a regatta. Nutrient intake during this time offers lightweight rowers an opportunity to recover, at least partially, from the physiological effects of any acute weight loss strategies undertaken prior to weigh-in. The intakes of fluid, electrolytes and carbohydrate are particularly important during this time; the focus of recovery strategies should be adjusted to reflect the method/s of acute weight loss implemented.

For example, aggressive rehydration strategies would be a priority if acute weight loss was achieved by inducing a state of hypohydration, while more aggressive carbohydrate intake would be warranted if weight loss were achieved by energy restriction. In the series of investigations undertaken by our group exploring the performance implications of acute weight 
loss, a recovery meal was formulated (toasted bread, Vegemite, Power Bar, Carboshotz, Gastrolyte, Gatorade, water), in accordance with the existing scientific literature, that was used and well tolerated across in excess of 100 performance tests. The meal achieved the following nutrient profile:

- $44.8 \mathrm{~kJ} / \mathrm{kg}$

- $2.3 \mathrm{~g} / \mathrm{kg}$ carbohydrate

- $33 \mathrm{mg} / \mathrm{kg}$ sodium

- $28.5 \mathrm{~mL} / \mathrm{kg}$ fluid

Research suggests that lightweight rowers should be encouraged to maximise recovery in the $12-16 \mathrm{~h}$ following racing in an attempt to optimise subsequent performance, even when they have to weigh-in again $48 \mathrm{~h}$ later, and thus likely be required to implement acute weight loss strategies in the $24 \mathrm{~h}$ before weigh-in. As with other competition strategies, recovery strategies, including food and fluid intake, should be trialled in training to confirm tolerance prior to implementing in competition.

\section{JUNIOR ROWING}

Dr Tomislav Smoljanovic: Member of FISA Sports Medicine Commission. Olympic Bronze medallist (Sydney 2000), Croatian Rowing Federation.

What are the particular medical issues facing the musculoskeletal system of adolescent/junior rowers?

Adolescence is a phase that separates early childhood and adulthood during which children go through a number of physical and psychological transitions while they mature. Usually children start rowing during or just before the onset of adolescence and by the end of it (approximately under the age of 19 years) these young (junior) rowers use the same rowing equipment, compete at the same race distance, and train with similar frequency and duration as elite senior rowers.

Junior rowers have a higher annual aggregate injury rate $(0.35$ injuries per 1000 training sessions or more) than senior rowers. Some of these injuries are caused by lack of rowing experience as evidence shows that those injured during on-water training have rowed for a shorter time than the rowers who did not sustain injuries. Also, there is a significantly higher incidence of traumatic low back injuries among sweep rowers who changed rowing side during the current season. Acute injuries in junior rowers (compared with senior rowers) may be caused by lack of experience among rowing coaches as evidence indicates that inappropriate training may be a risk factor.

Training volume is significantly associated with injury in junior rowers and those who average more than seven training sessions per week during a rowing season are at higher risk of more total injuries and more overuse low back injuries compared with those with fewer training sessions per week. Recent findings of Carsen et al found that a cam deformity was present exclusively in the closed physeal group of junior rowers who were screened, strongly supporting development of the deformity during the period of physeal closure (adolescence) with increased activity level as a possible risk factor. Cam deformity can cause damage to hips' soft tissues (cartilage, labrum, etc); Boykin et al have shown that $33 \%$ of 18 rowers surgically treated for the condition never returned to rowing. This deformity (if symptomatic and left untreated) may lead to early onset of hip osteoarthritis requiring total joint replacement sometimes even in patients in their early 30 s.

Considering the injury profile, should training for junior rowers differ from seniors?

Coaches of young rowers must consider that these athletes have not completed their growth and maturation; thus they have open physeal plates, which are comprised of cartilage tissue that in some of the rowers cannot withstand supraphysiological stresses placed by too frequent and/or too intense training. Training sessions must be adjusted by volume, intensity and frequency to suit the individual condition of the young rowers. Furthermore, open physeal plates elongate bones faster than soft tissue causing stress on muscle attachment points (also often made of cartilage at this age) and joints. Adoption of proper and regular stretching programmes that are performed after each training or race may reduce injury risk. Elongation of the limbs causes proprioception problems that, in addition to weakening the muscles, make young rowers more prone to different acute injuries. Avoidance of complex cross-training should be avoided in this group.

Competing interests None.

Provenance and peer review Not commissioned; internally peer reviewed.

\section{REFERENCE}

1 Drezner JA, Ackerman MJ, Anderson J, et al. Electrocardiographic interpretation in athletes: the 'Seattle Criteria'. Br J Sports Med 2013;47:122-4. 

clinical conundrums

Fiona Wilson and Alison McGregor

Br J Sports Med 2014 48: 1525-1528

doi: $10.1136 /$ bjsports-2014-094246

Updated information and services can be found at:

http://bjsm.bmj.com/content/48/21/1525

\begin{abstract}
These include:
References This article cites 1 articles, 1 of which you can access for free at: http://bjsm.bmj.com/content/48/21/1525\#BIBL

Email alerting

Receive free email alerts when new articles cite this article. Sign up in the service box at the top right corner of the online article.
\end{abstract}

\title{
Notes
}

To request permissions go to:

http://group.bmj.com/group/rights-licensing/permissions

To order reprints go to:

http://journals.bmj.com/cgi/reprintform

To subscribe to BMJ go to:

http://group.bmj.com/subscribe/ 